\title{
The Effect of $N$-Ethylmaleimide on the Radiation Sensitivity of Bacteria
}

\author{
By B. A. BRIDGES \\ Isotope Research Division, Wantage Research Laboratory \\ (A.E.R.E.), Wantage, Berkshire
}

(Received 28 March 1961)

\begin{abstract}
SUMMARY
The sensitization of Escherichia coli strain $\mathrm{B} / \mathrm{r}$ to $\gamma$ radiation was shown to take place within a few minutes of the addition of $N$-ethylmaleimide. Sensitization was demonstrated with as little as $0.0001 \mathrm{M}-N$-ethylmaleimide, but a much higher concentration ( $>0.002 \mathrm{M}$ ) was necessary for any bactericidal effect. The $N$-ethylmaleimide had to be present during irradiation in order to be effective. Staphylococcus aureus and a Pseudomonas sp. but not spores of Bacillus subtilis, were also sensitized by $N$-ethylmaleimide. The Pseudomonas sp. was only sensitized under anoxic conditions; this may indicate that, with this organism, $N$-ethylmaleimide and oxygen acted competitively in enhancing radiation damage. Possible mechanisms of sensitization are discussed.
\end{abstract}

\section{INTRODUCTION}

Nitric oxide and oxygen both enhance radiation damage when present in suspensions of the vegetative forms of bacteria during irradiation (Howard-Flanders, 1957; Hollaender, Stapleton \& Martin, 1951). Oxygen is also effective with spores, although nitric oxide has been found to have a net protective effect on them (Powers, Webb \& Kaleta, 1960). Until recently little attention had been paid to other substances which might sensitize bacteria to radiation. Bridges (1960) reported that $N$-ethylmaleimide (NEM), when present during irradiation, was able to increase the lethal action of $\gamma$ radiation on Escherichia coli, an effect more pronounced under anoxic conditions. The present paper records further study of the action of NEM on certain micro-organisms.

\section{METHODS}

Chemical. $N$-ethylmaleimide (NEM) was obtained from the Aldrich Chemical Co. Inc. (Milwaukee, Wisconsin, U.S.A.). It was dissolved in sterile 0.067 M-phosphate buffer ( $\mathrm{pH} \mathrm{7)}$ to make a $0.01 \mathrm{M}$-solution which was kept at $3-5^{\circ}$ and diluted as required. Sterilization of this solution was found to be unnecessary.

Micro-organisms. Escherichia coli strain B/r (in the sequel this strain will be referred to as Escherichia coli B/r), obtained in 1958 from Miss Tikvah Alper (Hammersmith Hospital), was used throughout this work. The Pseudomonas sp. was isolated from a chicken carcass by Dr Margaret Thornley (Low Temperature Research Station, Cambridge), and Staphylococcus aureus was no. 7447 of the National Collection of Type Cultures. Cultures (20-24 hr.) on nutrient agar slopes 
were washed off and shaken with $10 \mathrm{ml}$. 0.067 $\mathrm{M}$-phosphate buffer $(\mathrm{pH} 7)$ and $1 \mathrm{ml}$. of this suspension added to $9 \mathrm{ml}$. of the test solution before irradiation. Spores of Bacillus subtilis were obtained by allowing $24 \mathrm{hr}$. cultures on nutrient agar plates to stand on the bench for several days. The growth was then washed off with buffer, centrifuged, resuspended in buffer, and the suspension heated in an $80^{\circ}$ water bath for $10 \mathrm{~min}$. The spore suspensions were shaken with glass beads to break up clumps and were used within a few hours of preparation. NEM was added 4-6 min. before irradiation except where otherwise stated.

Estimation of radiation damage. The ability of bacteria to form visible colonies on the surface of nutrient agar (Oxoid) plates incubated at $30^{\circ}$ was used as the criterion of viability. Colonies usually appeared within $24 \mathrm{hr}$. although after treatment with a high concentration of NEM subsequent growth was slow and colonies continued to appear for up to six days.

Irradiation technique. $\mathrm{A}^{60} \mathrm{Co} \gamma$ radiation source giving a dose rate of $300 \mathrm{krad} . / \mathrm{hr}$. was used at room temperature. Samples $(10 \mathrm{ml}$.) of test suspension were irradiated in glass vessels, and air or oxygen-free nitrogen was bubbled vigorously through the suspensions during the irradiation. With nitrogen, bubbling was begun $6 \mathrm{~min}$. before irradiation, to remove dissolved oxygen.

\section{RESULTS}

Sensitization of Escherichia coli $\boldsymbol{B} / \boldsymbol{r}$

The survival curves for Escherichia coli B/r in the presence and absence of $0.001 \mathrm{M}$ NEM are shown in Fig. 1; it can be seen that a pronounced sensitization occurred. Where the shape of survival curves of sensitized and control bacteria is the same, the extent of any sensitization may be described by the 'dose modifying factor' (d.m.f.) which is the ratio of the dose of radiation needed to cause a given degree of damage in the absence of the sensitizer to that required in its presence. For example, the presence of oxygen in an air-saturated suspension results in a d.m.f. of $2 \cdot 6$ with $E$. coli B/r. Under anoxic conditions, $0.001 \mathrm{M}-\mathrm{NEM}$ was equivalent to a d.m.f. of $\mathbf{2} \cdot \mathbf{0}$, but sensitization was not so marked under aerated conditions where the d.m.f. was $1 \cdot 3$. There was no enhancement of radiation damage when NEM (to $0.001 \mathrm{M}$ ) was added immediately after irradiation in buffer (Fig. 1). Bacteria initially incubated with $0.0005 \mathrm{M}-\mathrm{NEM}$ for $5 \mathrm{~min}$. and then diluted 100 -fold or treated with

Table 1. The effect of removing N-ethylmaleimide (NEM) immediately before irradiation on the radiation sensitivity of Escherichia coli strain $B / r$

NEM was used at $0.0005 \mathrm{M}$; irradiations were performed under anoxic conditions.

Mean survival after $30 \mathrm{krad}$.

$(\%)$

Untreated controls

$33 \cdot 1$

NEM added $10 \mathrm{~min}$. before irradiation

(a) Not removed

$16 \cdot 1$

(b) Removed by addition of excess

$31 \cdot 6$ cysteine $5 \mathrm{~min}$. before irradiation

(c) Removed by 1/100 dilution 5 min. before irradiation 
excess $(0.001 \mathrm{M})$ cysteine to remove any unreacted NEM were not sensitized to subsequent irradiation in absence of oxygen (Table 1).

The greater effect of NEM under anoxic conditions might have been due to a requirement for a period of incubation with the compound before irradiation, since under anoxic conditions there was always nitrogen bubbling for $6 \mathrm{~min}$. before each incremental dose of radiation. Various times of pre-incubation under aerobic conditions were therefore tried. It can be seen from Fig. 2 that a definite sensitization was observed with pre-incubation for as little as $1 \mathrm{~min}$. There was no increase in sensitization when the pre-incubation period was increased from 5 to $15 \mathrm{~min}$. The difference in pre-incubation time does not therefore explain the difference in effect as between aerated and anoxic conditions. The rapidity with which sensitization was established would seem to exclude the possibility that any gross changes in the physiological state of the cells before irradiation were involved although it does not exclude the possible involvement of the reaction with cellular $-\mathrm{SH}$ groups.

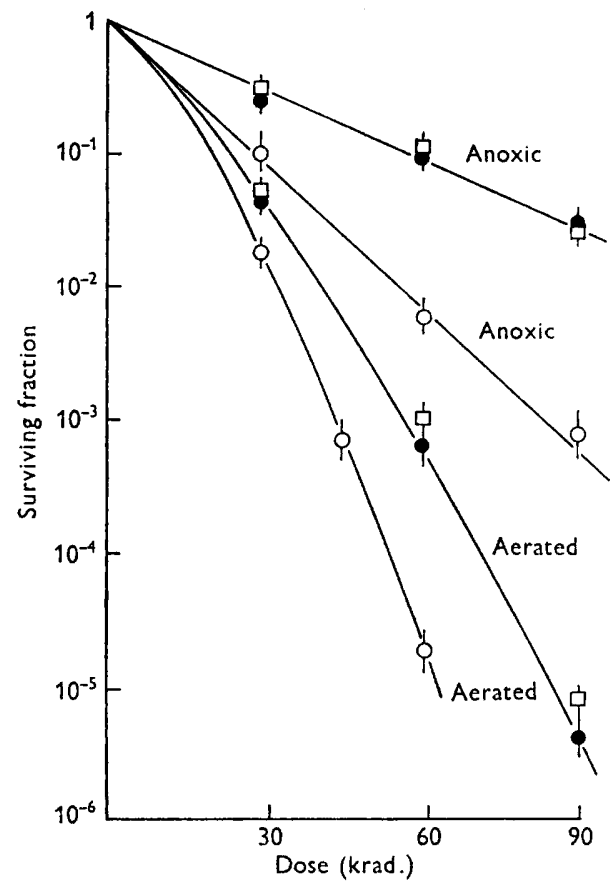

Fig. 1

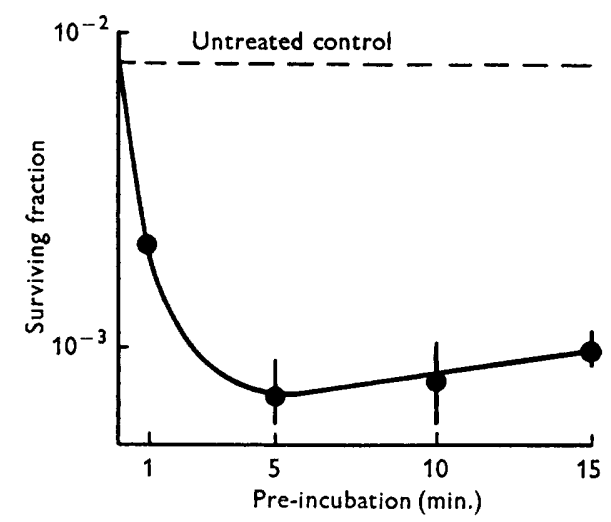

Fig. 2

Fig. 1. Effect of $0.001 \mathrm{M}-\mathrm{NEM}$ on the radiation sensitivity of Escherichia coli strain B/r. $\odot$, No NEM; O, NEM present during irradiation; $\square$, NEM added immediately after irradiation.

Fig. 2. Effect of pre-incubation with $0.001 \mathrm{M}-\mathrm{NEM}$ on surviving fraction of Escherichia coli strain $B / \mathrm{r}$ after $45 \mathrm{krad}$. under aerated conditions.

\section{Toxicity of NEM}

Figure 3 shows the toxicity of NEM at varying concentrations. As judged by the ability of the treated organisms to form visible colonies on nutrient agar, NEM was non-toxic up to $0.001 \mathrm{M}$, while at $0.01 \mathrm{M}$ there was an appreciable lethal effect. Although treatment with $0.001 \mathrm{~m}-\mathrm{NEM}$ for $1 \mathrm{hr}$. did not prevent the subsequent 
formation of colonies, they appeared more slowly than the controls. Colonies appeared at the normal rate, however, when $0.001 \mathrm{M}$-homocysteine was present in the plating medium. The presence of this sulphydryl compound did not decrease the toxicity of NEM or the degree of sensitization.

\section{Effect of concentration}

The influence of concentration on sensitization under anoxic conditions is given in Fig. 4; a similar curve was obtained under aerated conditions. There appears to be a threshold at about $0.00005 \mathrm{M}-\mathrm{NEM}$, below which no significant sensitization was observed. Above this concentration the effect increased rapidly and reached a maximum at $0.0003 \mathrm{M}$. Above this there was apparently a very slight decrease in sensitization.

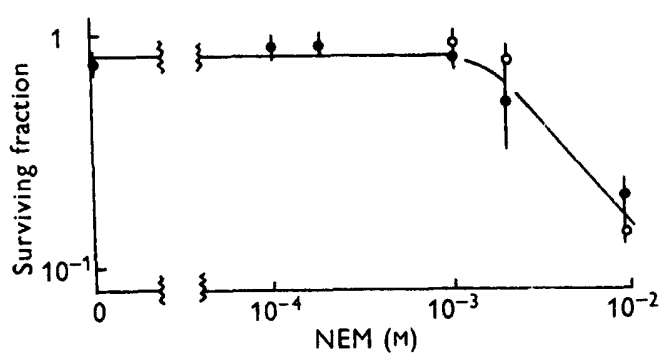

Fig. 3

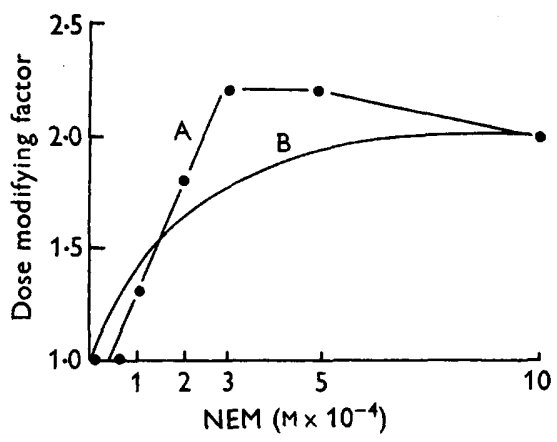

Fig. 4

Fig. 3. Effect of incubation with NEM for $1 \mathrm{hr}$. on viability of Escherichia coli strain B/r. e, Plate counts on nutrient agar; $\mathrm{C}$, Plate counts on nutrient agar containing $0.001 \mathrm{M}$ homocysteine.

Fig. 4. Effect of concentration of NEM on surviving fraction of Escherichia coli strain $B / \mathrm{r}$ after $60 \mathrm{krad}$. under anoxic conditions. A, experimental curve; $B$, theoretical curve according to equation of Alper \& Howard-Flanders (1956) where $m=2 \cdot 2, K=175 \mu \mathrm{M}$.

Alper \& Howard-Flanders (1956), in studying the oxygen effect, represented the effect of concentration by the following formula:

$$
\text { d.m.f. }=\frac{m\left[\mathrm{O}_{2}\right]+K}{\left[\mathrm{O}_{2}\right]+K},
$$

where $m$ is the maximum d.m.f. and $K$ is the concentration of oxygen at which half the maximum sensitization is attained. Applying these terms to NEM under anoxic conditions, $m$ may be assumed to be $2 \cdot 2$ and $K, 175 \mu \mathrm{M}$. A curve plotted from these values in such an equation is shown in Fig. 4; the curve does not characterize the experimental points. The effect of the concentration of NEM appears therefore to be significantly different from that of oxygen.

\section{Experiments with other organisms}

The effect of 0.001 M-NEM on the radiation sensitivity of Staphylococcus aureus is shown in Fig. 5. It was essentially similar to that obtained with Escherichia coli $\mathrm{B} / \mathrm{r}$, the d.m.f. values being 1.55 under anoxic conditions and 1.17 in air. The Pseudomonas sp. differed from $S$. aureus and $E$. coli $\mathrm{B} / \mathbf{r}$ in that although no sensitization was produced by $0.001 \mathrm{M}-\mathrm{NEM}$ under aerated conditions, there was a very marked effect 
under anoxic conditions where the d.m.f. was $2 \cdot 0$ (Fig. 6). It is of some interest that the effect of oxygen on radiation sensitivity was large with this pseudomonad, the slope of the survival curve under air being 4.25 times as great as that under nitrogen. Spores of Bacillus subtilis were not sensitized to radiation by $0.001 \mathrm{M}-\mathrm{NEM}$ under anoxic or aerated conditions. This might have been due to the failure of NEM to penetrate the spore wall but no experiments were carried out to verify this. NEM was not toxic to any of the above organisms at $0.001 \mathrm{M}$.

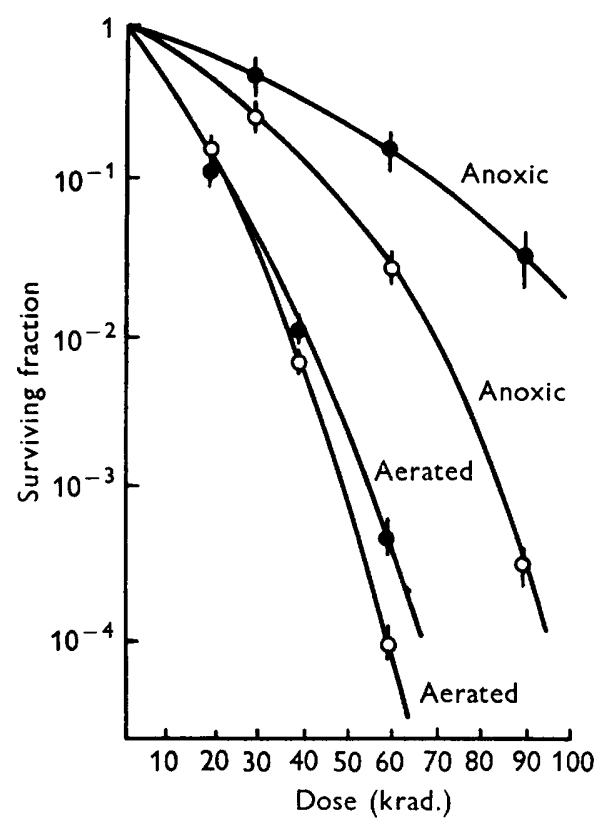

Fig. 5

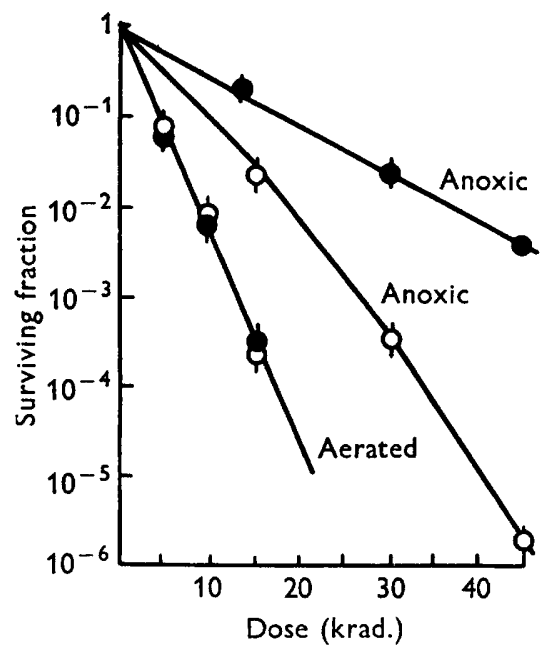

Fig. 6

Fig. 5. Effect of $0.001 \mathrm{~m}-\mathrm{NEM}$ on the radiation sensitivity of Staphylococcus aureus. , Control; O, NEM.

Fig. 6. Effect of $0.001 \mathrm{M}-\mathrm{NEM}$ on the radiation sensitivity of Pseudomonas sp. , Control; O, NEM.

\section{DISCUSSION}

Sensitization is apparently dependent upon the simultaneous action of NEM and radiation since organisms incubated with $0.0005 \mathrm{M}-\mathrm{NEM}$ for $10 \mathrm{~min}$. and then diluted $1 / 100$ or treated with excess cysteine (to $0.001 \mathrm{M}$ ) to remove unreacted NEM, were not sensitized. This eliminates the two most obvious explanations of the mechanism of sensitization, i.e. that NEM combines with cellular -SH groups and in some way renders them more sensitive to the action of radiation, or that NEM combines with naturally occurring - SH protective agents. Sulphydryl groups on biological molecules have been classified as 'freely reacting', 'sluggish' and 'masked' (Barron, 1951). All the freely reacting -SH groups are likely to react with NEM within a very few minutes of its addition (Roberts \& Rouser, 1958), and since the reaction is irreversible they will lose their biological activity. That some physiological damage does occur seems indicated by the finding that the endogenous respiration of Escherichia coli $\mathrm{B} / \mathrm{r}$ is $70 \%$ inhibited by $0.001 \mathrm{M}-\mathrm{NEM}$ (Bridges \& Marples, un- 
published observation). This damage to cellular -SH groups is obviously not lethal since the organisms are able to form colonies when diluted out and plated. Although colony formation is slower than usual it may be speeded by up the addition of - SH groups to the medium in the form of homocysteine. Such a treatment does not, however, overcome the sensitization.

The fact that a certain amount of NEM reacts with cellular -SH groups probably accounts for the threshold observed in the concentration curve. Presumably a certain concentration is required before there is sufficient uncombined NEM remaining to cause sensitization. The organisms used in these experiments were not washed and might be expected to have had a fairly high concentration of - $\mathrm{SH}$ compounds arising from the nutrient medium, in addition to those normally present within the organism.

A possible explanation of the data is that the NEM reacts with some molecule which is in a short-lived reactive state induced by the radiation. This molecule, in the absence of NEM, stands a certain chance of being restored or changed to an inocuous form. A similar model has been proposed for the enhancing effect of oxygen (Alper \& Howard-Flanders, 1956; Alper, 1958). In this case the oxygen is envisaged as undergoing a 'metionic' reaction with a free radical centre, probably on a carbon atom, resulting in the formation of a peroxide radical. It is quite possible that NEM could react across its double bond with a carbon-free radical centre. Such reactions are known in the radiation-induced cross-linking of rubber which is catalysed by dimaleimides (Vale \& Roberts, 1960). A further possibility is that NEM reacts with -SH groups (or possibly -S-free radicals) which result from the radiation-induced breakage of -S-S-bonds, necessary for the functional state of some proteins and other molecules of biological importance. Combination of NEM with either of the sulphur atoms could prevent the -S-S- bond reforming and result in the loss of the biological integrity of the molecule.

Since oxygen partially (completely, with the Pseudomonas sp.) inhibited the action of NEM, it is conceivable that both compete for the same free radical centres. The study of sensitizing agents such as NEM may therefore be of use in understanding the oxygen effect and, ultimately, the nature of the lethal damage which radiation causes within the cell.

\section{REFERENCES}

Alper, T. (1958). A mechanism for the oxygen effect suggested by some recent experiments. In Organic Peroxides in Radiobiology. Ed. R. Latarjet. Paris: Masson et Cie.

Alper, T. \& Howard-Flanders, P. (1956). Role of oxygen in modifying the radiosensitivity of $E$. coli B. Nature, Lond. 178, 979.

Barron, E. S. G. (1951). Thiol groups of biological importance. Advanc. Enzymol. 11, 201.

Bridges, B. A. (1960). Sensitization of Escherichia coli to gamma radiation by $N$-ethylmaleimide. Nature, Lond. 188, 415.

Hollaender, A., Stapleton, G. E. \& Martin, F. L. (1951). X-ray sensitivity of E. coli as modified by oxygen tension. Nature, Lond. 167, 103.

Howard-Flanders, P. (1957). Effect of nitric oxide on the radiosensitivity of bacteria. Nature, Lond. 180, 1191.

Powers, E. L., WebB, R. B. \& Kaleta, B. F. (1960). Oxygen and nitric oxide as modifiers of radiation injury in spores of Bacillus megaterium. Proc. nat. Acad. Sci., Wash. 46, 984.

Roberts, E. \& Rouser, G. (1958). Spectrophotometric assay for reaction of $\mathrm{N}$-ethylmaleimide with sulphydryl groups. Anal. Chem. 30, 1291.

VALE, R. L. \& RoBERTs, R. (1960). Use of maleimides to reduce radiation dose necessary to cross-link hydrocarbon polymers. British Patent Application P16712/60. 